\title{
CCR3 is essential for skin eosinophilia and airway hyperresponsiveness in a murine model of allergic skin inflammation
}

\author{
Weilie Ma, ${ }^{1}$ Paul J. Bryce,${ }^{1}$ Alison A. Humbles, ${ }^{2}$ Dhafer Laouini,${ }^{1}$ Ali Yalcindag, ${ }^{1}$ \\ Harri Alenius, ${ }^{1}$ Daniel S. Friend, ${ }^{3}$ Hans C. Oettgen, ${ }^{1}$ Craig Gerard, ${ }^{2}$ and Raif S. Geha ${ }^{1}$ \\ ${ }^{1}$ Division of Immunology, and Department of Pediatrics, \\ ${ }^{2}$ Division of Pulmonary Medicine and Ina Sue Perlmutter Laboratory, Children's Hospital, and \\ ${ }^{3}$ Department of Pathology, Brigham and Women's Hospital, Harvard Medical School, Boston, Massachusetts, USA
}

Address correspondence to: Raif S. Geha, Enders 8, Division of Immunology, Children's Hospital, 300 Longwood Avenue, Boston, Massachusetts 02115, USA. Phone: (617) 355-7602; Fax: (617) 355-8205; E-mail: raif.geha@tch.harvard.edu.

Received for publication November 27, 2001, and accepted in revised form December 10, 2001.

\begin{abstract}
The CC chemokine receptor 3 (CCR3) is expressed by eosinophils, mast cells, and Th2 cells. We used $\mathrm{CCR}^{-/-}$mice to assess the role of CCR3 in a murine model of allergic skin inflammation induced by repeated epicutaneous sensitization with ovalbumin (OVA), and characterized by eosinophil skin infiltration, local expression of Th2 cytokines, and airway hyperresponsiveness (AHR) to inhaled antigen. Eosinophils and the eosinophil product major basic protein were absent from the skin of sham and OVA-sensitized CCR3 $3^{--}$mice. Mast cell numbers and expression of IL-4 mRNA were normal in skin of CCR3-/- mice, suggesting that CCR3 is not important for infiltration of the skin by mast cells and Th2 cells. CCR3-/- mice produced normal levels of OVA-specific IgE, and their splenocytes secreted normal amounts of IL-4 and IL-5 following in vitro stimulation with OVA, indicating effective generation of systemic Th2 helper responses. Recruitment of eosinophils to lung parenchyma and bronchoalveolar lavage (BAL) fluid was severely impaired in CCR3-/- mice, which failed to develop AHR to methacholine following antigen inhalation. These results suggest that CCR3 plays an essential role in eosinophil recruitment to the skin and the lung and in the development of AHR.
\end{abstract}

J. Clin. Invest. 109:621-628 (2002). DOI:10.1172/JCI200214097.

\section{Introduction}

Atopic dermatitis (AD) is a common pruritic inflammatory skin disease that often begins in infancy and frequently affects individuals with personal or family history of atopic disease. The majority of infants with $\mathrm{AD}$ develop asthma and/or allergic rhinitis later in life (1). The histology of $\mathrm{AD}$ skin lesion reveals a marked inflammatory cell infiltrate that consists of eosinophils, lymphocytes, monocytes/macrophages, and Langerhans cells. In chronic lesions there are also increased numbers of mast cells (1). Lymphocytes infiltrating the skin lesions of $\mathrm{AD}$ are predominantly $\mathrm{CD}^{+}, \mathrm{CD}^{+}$, and $\mathrm{CD}_{45 \mathrm{RO}^{+}}$memory T cells (2). The Th2 cytokines (IL-4, IL-5, and IL-13) are expressed in acute skin lesions of $\mathrm{AD}$, whereas the Th1 cytokine IFN- $\gamma$ is found in later stages of the disease $(3,4)$.

IL-5 stimulates the maturation of eosinophils from $\mathrm{CD} 34^{+}$precursor cells in the bone marrow and their release into the circulation (5). IL-5 also primes eosinophils for responsiveness to chemotactic factors (6). Increased numbers of circulating eosinophils have been observed in patients with $\mathrm{AD}$ (7). Furthermore, programmed cell death of peripheral blood eosinophils is delayed in $\mathrm{AD}$ (8). Although intact eosinophils are sparse in $\mathrm{AD}$ lesional skin, eosinophil granule proteins, i.e., eosinophil-derived major basic protein (MBP) and eosinophil cationic protein, are increased in the periph- eral blood of patients with $\mathrm{AD}$ and correlate with disease activity (9). Moreover, these proteins are also deposited in lesional skin of $\mathrm{AD}(10,11)$. These data indicate that eosinophil activation and degranulation occurs in $\mathrm{AD}$.

Chemokines play an important role in the infiltration of inflammatory cells into tissues. Chemokines are classified into four subclasses (CC, CXC, C, and CX3C), based on the location of the first two cysteines in their sequence. The biological effects of chemokines are mediated by their interaction with specific receptors that belong to the seven-transmembrane G-protein-coupled receptors (12). The principal receptor involved in eosinophil attraction is CCR3 (13), which is also expressed by Th2 cells and mast cells $(14,15)$. The major ligands for CCR3 are eotaxin, eotaxin-2, eotaxin-3, monocyte chemoattractant protein-2 (MCP-2), MCP-3, MCP-4, and RANTES (16). The association of CCR 3 and its ligands with asthma has been studied both in patients and in murine models of asthma. Eotaxin and CCR3 mRNA are expressed and colocalized in the bronchial mucosa of asthma patients. Moreover, the intensity of their expression correlates with increased airway hyperresponsiveness (AHR) (17). Furthermore, allergen challenge in patients with allergic asthma causes upregulation of eotaxin expression in the bronchial mucosa and RANTES expression in 
bronchoalveolar lavage (BAL) fluid (16). In an ovalbumin-induced (OVA-induced) mouse asthma model, administration of neutralizing Ab's to eotaxin, or MCP-3, or of the receptor antagonist met-RANTES partially reduced $\mathrm{T}$ cell and eosinophil infiltration and AHR $(18,19)$. Furthermore, eotaxin-deficient mice have $70 \%$ less eosinophils in BAL fluid 18 hours after airway antigen challenge (20). Taken together, these studies suggest an important role for CCR 3 and its ligands in recruiting eosinophils to the lung and in the subsequent development of AHR.

Eotaxin, MCP-3, and RANTES, as well as CCR3, are expressed in human AD skin lesions (21-23). However, the importance of CCR3 and its ligands in AD has not been examined. We have reported that eotaxin, MCP-3, and RANTES are also expressed in OVA-sensitized skin sites in a murine model of allergic skin inflammation induced by repeated epicutaneous sensitization with OVA $(24,25)$. This model has many similarities to human AD including elevated total and specific IgE, as well as dermatitis characterized by infiltration of $\mathrm{CD}^{+} \mathrm{T}$ cells and eosinophils, and by local expression of mRNA for the cytokines IL-4, IL-5, and IFN- $\gamma$. Furthermore, mice epicutaneously sensitized to OVA and then challenged once with aerosolized OVA exhibit increased AHR to inhaled methacholine (Mch), a hallmark of asthma (26). We took advantage of the recent availability of CCR3-deficient mice to examine the role of CCR3 in our murine model of allergic skin inflammation. The results obtained suggest that CCR3 is essential for eosinophil recruitment into the skin at sites of antigen sensitization, as well as for eosinophil recruitment into the lung and the development of AHR following inhaled antigen challenge of epicutaneously sensitized mice.

\section{Methods}

Mice and sensitization. The CCR3-- mice were generated as described (27). CCR3-/- mice are of $129 / \mathrm{BALB} / \mathrm{c}$ background and have normal $\mathrm{T}$ and $\mathrm{B}$ cell phenotype and normal hematologic parameters, including leukocyte differential counts, platelets, and hematocrit. Ageand weight-matched $\mathrm{CCR} 3^{+/+}$littermates are used as wild-type (WT) controls. These animals were kept in a pathogen-free environment. All procedures performed on the mice were in accordance with the Animal Care and Use Committee of the Children's Hospital.

Epicutaneous sensitization of 4- to 6-week-old female mice was performed as described previously (24). Briefly, mice were anesthetized with Avertin (SigmaAldrich, Milwaukee, Wisconsin, USA) and then shaved with an electric razor. The shaved area was tape stripped three times by transparent dressing Tegaderm (Owens \& Minor Inc., Franklin, Massachusetts, USA) to remove loose hair and to introduce standardized skin injury as a surrogate for the excoriation induced by scratching in patients with $\mathrm{AD}$. One hundred micrograms of OVA (Grade V; Sigma Chemical Co., St. Louis, Missouri, USA) in $100 \mu$ l of normal saline or sham
(100 $\mu$ l of normal saline) were placed on a patch of sterile gauze $(1 \times 1 \mathrm{~cm})$, which was secured to the skin with Tegaderm. The patches were placed for a 1-week period and then removed. Two weeks later, an identical patch was reapplied to the same skin site. Each mouse had a total of three 1-week exposures to the patch separated from each other by 2-week intervals.

Serum IgE. Mice were bled and sera collected following third epicutaneous sensitization. The standard PharMingen (San Diego, California, USA) protocol for sandwich ELISA was used to quantify OVA-specific $\operatorname{IgE}$ Ab's (24). Results of OVA-specific IgE were expressed as nanograms per milliliter by comparison with a standard consisting of purified mouse OVAspecific IgE secreted by the hybridoma TOE, a kind gift of Mamoru Kiniwa (Immunology Research Laboratory, Hanno Research Center, Taiho Pharmaceutical Co. Ltd., Saitama, Japan) (28).

Histological analysis. For histological examination, specimens were obtained from patched areas on the skin following the third sensitization. Specimens were fixed in $10 \%$ buffered-formalin and embedded in paraffin. Multiple $4-\mu \mathrm{m}$ sections were stained with hematoxylin and eosin. Individual inflammatory cell types were counted blinded in 20 high-power fields (HPFs) at $\times 1,000$ and expressed as cells per HPF, with the mean calculated. Mast cells were identified in tissue sections after staining with toluidine blue. To identify eosinophils in the lung, lung sections were stained with Congo red, which stains eosinophils orange. Mast cells in the trachea were identified by staining trachea with a solution containing naphthol AS-D chloroacetate (29).

Immunohistological analysis. Skin sections were embedded in Tissue-Tek oxacalcitriol compound (Miles Inc., Elkhart, Indiana, USA) on dry ice. Sections of $4 \mu \mathrm{m}$ were prepared and were stained by an avidin-biotin method as described previously (30). Rabbit anti-MBP Ab is a kind gift from James Lee (Mayo Clinic, Scottsdale, Arizona, USA) (31). Endogenous peroxidase was blocked with $\mathrm{PBS} / 0.3 \% \mathrm{H}_{2} \mathrm{O}_{2}$ routinely, and staining without primary $\mathrm{Ab}$ was used as a negative control to ensure that brown cells are anti-MBP immunoreactive cells.

$R N A$ preparation and PCR amplification of reverse-transcribed $c D N A$. Skin biopsies and lung samples were obtained at the end of the third sensitization and were immediately frozen in dry ice. To extract the RNA, the samples were homogenized in TRIzol (Life Technologies Inc., Grand Island, New York, USA) using a Polytron RT-3000 (Kinematica AG, Littau-Luzem, Switzerland). Further RNA extraction was performed following the manufacturer's instruction. The cDNA was synthesized from $10 \mu \mathrm{g}$ of total RNA in a $40-\mu \mathrm{l}$ reaction mix using Superscript II (Life Technologies Inc.) following the manufacturer's instruction. The primers used to amplify cDNA for $\beta 2$-microglobulin, IL-4, IFN- $\gamma$, and DNA amplification were as described previously $(24,32$, 33). To quantify mRNA, a fixed amount of reverse-transcribed cellular mRNA was coamplified in the presence of serial dilutions of a multispecific internal plasmid 
control (pMUS3; ref. 32), which contains nucleotide sequences of multiple cytokines. The dilution of which pMUS3-derived and cDNA-derived signals were of equivalent intensity was used to establish the relative amount of cytokine. The results were expressed as a ratio of cytokine cDNA to cDNA of the constitutively expressed $\beta 2$-microglobulin gene.

In vitro IL-4 and IL-5 synthesis. Single cell suspensions of spleen cells were prepared from mice in complete RPMI-1640 (JRH Biosciences, Lenexa, Kansas, USA) supplemented by $10 \%$ FCS, $1 \mathrm{mM}$ sodium pyruvate, 2 $\mathrm{mM}$ L-glutamine, $0.05 \mathrm{mM}$ 2-ME, $100 \mathrm{U} / \mathrm{ml}$ penicillin, and $100 \mu \mathrm{g} / \mathrm{ml}$ streptomycin. Cells were cultured in the above medium at $2 \times 10^{6} / \mathrm{ml}$ in 24 -well plates in the presence of OVA $(50 \mu \mathrm{g} / \mathrm{ml})$. Supernatants were collected after 96 hours of culture, centrifuged, and frozen until use. IL-4 and IL-5 in supernatants were determined by ELISA following the manufacturer's instructions (PharMingen).

Analysis of eosinophils in peripheral blood. Blood was collected following the third sensitization. The absolute eosinophil count was determined by counting heparinized blood in a hemocytometer after staining with Discombe's fluid (34).

$B A L$. Immediately after sacrifice, cells in the lungs were recovered by flushing $0.8 \mathrm{ml}$ of BAL fluid $(1 \mathrm{mM}$ EDTA, 10\% FCS, PBS) into the lungs via the trachea. Total cell counts were determined and $100 \mu \mathrm{l}$ of fluid were cytospun onto glass slides using a Cytospin 3 centrifuge (Shandon Lipshaw, Pittsburgh, Pennsylvania, USA) (400 $g$ for 4 minutes). Differential cell counts were performed after staining with Diff-Quik Stain Set (Baxter Healthcare Corp., Miami, Florida, USA).

$A H R$ to $M c h$. Lung functions were determined 24 hours after exposure to aerosolized OVA ( $1 \%$ in saline for 20 minutes by ultrasonic nebulization). Enhanced pause (Penh) was measured using whole body plethysmography (BUXCO, Troy, New York, USA) as previously described (35). Baseline readings were taken and averaged for 5 minutes. Aerosolized saline or Mch in increasing concentrations ( 1 to $333 \mathrm{mg} / \mathrm{ml}$ ) were nebulized through an inlet of the main chamber for 2 minutes. Increases in airway resistance to aerosolized Mch were determined as Penh values, during and after the exposure (10-minute total analysis time).

Statistical analysis. Except for Penh, nonparametric Mann-Whitney tests were used to compare the different mice groups, since standard deviations varied widely between groups. Penh results were analyzed using two-way ANOVA. A $P$ value smaller than 0.05 was considered statistically significant.

\section{Results}

Eosinophils are virtually absent in OVA-sensitized skin sites of $\mathrm{CCR}^{-/-}$mice. Dermal infiltration with eosinophils is an important feature of our model of allergic skin inflammation elicited by epicutaneous allergen application (24). Few eosinophils were present in sham-sensitized skin from WT mice. This number significantly increased following epicutaneous sensitization with OVA. In contrast, no eosinophils were detected in shamsensitized skin from $C C R 3^{-/-}$mice. More importantly, eosinophils were virtually absent in the skin of CCR3--mice following OVA sensitization (Figure 1a).

The virtual absence of eosinophils in the skin of $\mathrm{CCR}^{-/-}$mice may have resulted from their failure to infiltrate and/or survive in the skin. The eosinophil product MBP has been used as a marker for eosinophils in tissues and remains detectable following eosinophil apoptosis (36). Sensitized skin sites from CCR3 $3^{--}$mice and WT controls were stained for MBP using immunoperoxidase. MBP staining was readily detectable in sham-sensitized skin sites and markedly increased following OVA sensitization in WT mice. In contrast, no MBP staining was detectable in either sham-sensitized or OVA-sensitized skin sites of $\mathrm{CCR}^{-/}$mice (Figure $1 \mathrm{~b}$ ).

One possible explanation for the absent eosinophils in the skin of $C C R 3^{-/}$mice is lack of eosinophil mobilization from the bone marrow into the blood. There was no statistically significant difference in the number of circulating eosinophils between CCR3-/- mice and WT con-

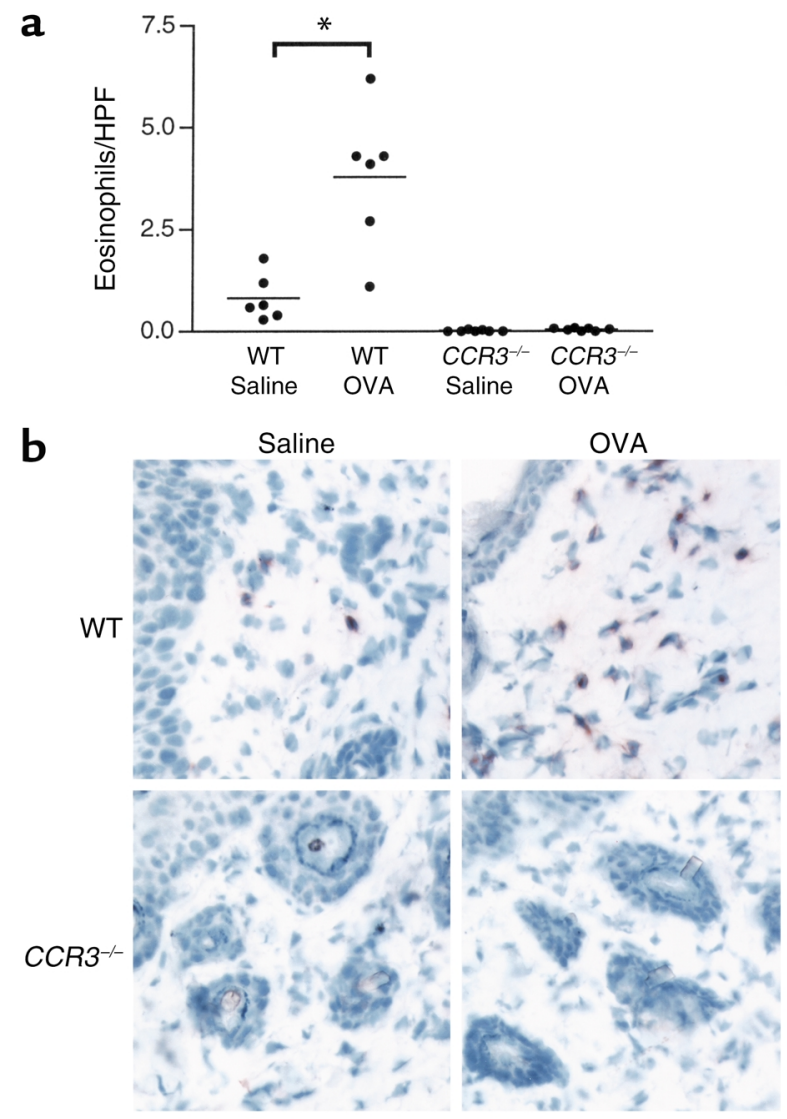

Figure 1

Eosinophils are virtually absent in OVA-sensitized skin sites of CCR3-/mice. (a) Eosinophils/HPF in skin sites of CCR3-/- mice and WT controls sensitized with either OVA or saline. The bars represent the mean ( $n=6-7$ animals per group). ${ }^{*} P<0.01$. (b) Immunoperoxidase MBP staining of sensitized skin sites from CCR3-/- mice and WT controls. MBP stains brown. 

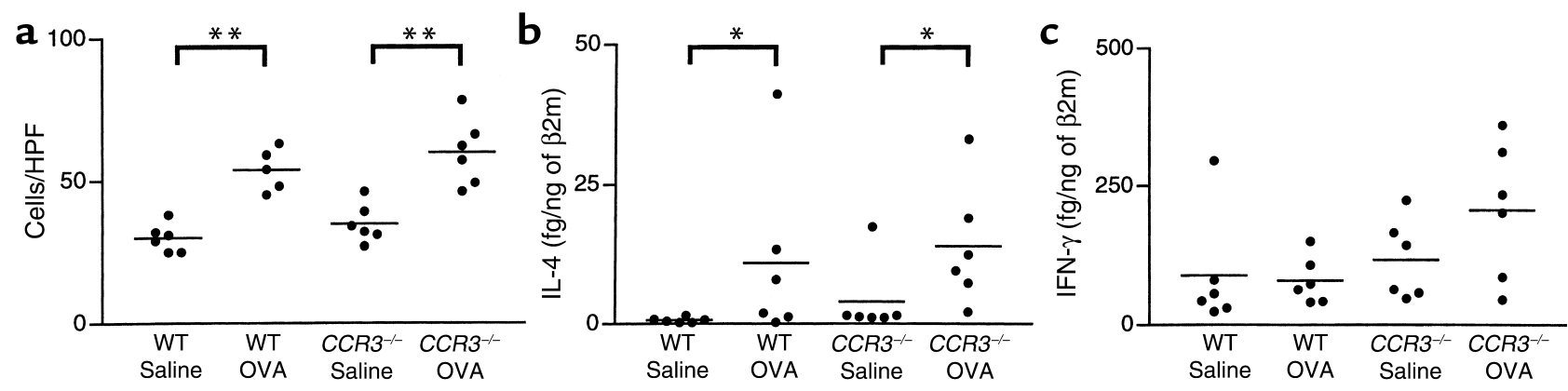

Figure 2

Total mononuclear cell counts in CCR3-/- mice are normal (a). CCR3-/- mice have normal expression of IL-4 (b) and IFN- $\gamma(\mathbf{c}) \mathrm{mRNA}$ in OVA-sensitized skin. Cytokine mRNA levels were normalized to $\beta 2$-microglobulin. The bars represent the mean ( $n=6$ animals per group). ${ }^{*} P<0.05 ;{ }^{*} P<0.01$

trols sensitized with either saline (WT: $0.74 \pm 0.21 \times 10^{5}$ eosinophils/ml; CCR3--: $1.38 \pm 0.46 \times 10^{5}$ eosinophils $/ \mathrm{ml}$ ) or OVA (WT: $1.75 \pm 0.32 \times 10^{5}$ eosinophils $/ \mathrm{ml}$; CCR3--: $1.83 \pm 0.58 \times 10^{5}$ eosinophils $/ \mathrm{ml}$ ).

OVA-sensitized skin of CCR3 $3^{-/-}$mice has normal numbers of mononuclear cells, expression of mRNA for Th2 (IL-4), and Th1 (IFN- $\gamma$ ) cytokines, and normal numbers of mast cells. OVA-sensitized skin sites in our model exhibit increased numbers of mononuclear cells, which consist of predominantly $\mathrm{CD}^{+} \mathrm{T}$ cells and macrophages. Furthermore, mRNA expression of the Th2 cytokine IL-4 is markedly increased in these sites, suggesting the presence of Th2 cells (24). Expression of mRNA for the Th1 cytokine IFN- $\gamma$ is modestly increased in some, but not all mouse strains tested (24, $25,37)$. Th2, but not Th1 cells, express CCR 3 (15) and migrate in response to an eotaxin gradient in vitro (38). It was therefore important to examine whether cellular infiltration and Th cytokine expression were affected by the absence of CCR3.

OVA sensitization resulted in a comparable increase in the number of mononuclear cells (Figure 2a) and of $\mathrm{CD}^{+} \mathrm{T}$ cells (data not shown) in the dermis of CCR3-/mice and WT controls. More importantly, OVA sensitization resulted in a marked and comparable increase in IL-4 mRNA expression in CCR $3^{-/-}$mice and WT controls (Figure 2b). There was no significant increase in the expression of IFN- $\gamma$ mRNA following OVA sensitization in either CCR3 $3^{-/-}$mice or WT controls (Figure 2c).

Mast cells are derived from bone marrow progenitors, which migrate to the peripheral tissues as immature cells and undergo differentiation in situ (39). Since mast cells express CCR3 $(40,41)$, we examined mast cells by toluidine blue staining. The number of total and degranulated mast cells were slightly elevated in OVA-sensitized skin compared with sham-sensitized skin in both WT mice and CCR $3^{-/}$mice, but the differences were not statistically significant (data not shown). There were no differences in the numbers of total and degranulated mast cells between WT and CCR3 $3^{-/}$mice (data not shown). These results suggest that CCR3 is not important for the homing and maturation of mast cell precursors into the skin.

Sensitized CCR $3^{-/-}$mice mount a normal Th2 response. Our murine model of allergic skin inflammation is characterized by a Th2-dominated systemic response characterized by elevated antigen-specific $\operatorname{IgE}(24)$ and by production of Th 2 cytokines by antigen-stimulated splenocytes (see Figure 3, a and b). Splenocytes from OVA-sensitized, but not from sham-sensitized, CCR3-- mice secreted IL-4 and IL-5 in amounts comparable to those secreted by WT controls (Figure 3, a and $b)$. The level of IFN- $\gamma$ production from splenocytes was not changed by sensitization and was com-
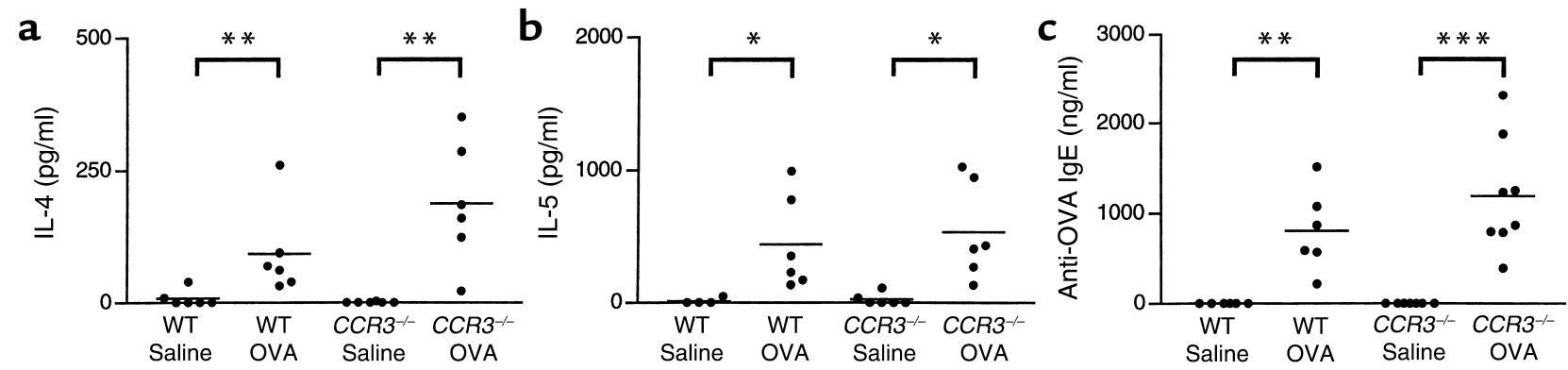

\section{Figure 3}

IL-4 (a) and IL-5 (b) in supernatants of splenocytes from CCR3-/- mice and WT controls sensitized with either OVA or saline following in vitro stimulation with OVA. The bars represent the mean ( $n=4-6$ animals per group). CCR $3^{-/-}$mice mount normal serum OVA-specific IgE (c) Ab responses. The bars represent the mean $\left(n=6-7\right.$ animals per group). ${ }^{*} P<0.05 ;{ }^{*} P<0.01 ;{ }^{*}{ }^{*} P<0.001$. 
parable in CCR3-/- mice and WT controls (data not shown). Furthermore CCR3-/- mice had levels of serum OVA-specific IgE comparable to WT controls (Figure $3 \mathrm{c}$ ). The level of serum OVA-specific $\operatorname{IgG}_{2 \mathrm{a}}$ was very low, but comparable in CCR3 $3^{--}$mice and WT controls (data not shown). These results suggest that CCR3 is not important for the Th2 response to epicutaneously applied antigen.

CCR3-/- mice epicutaneously sensitized with OVA do not develop BAL or lung tissue eosinophilia following inhalation challenge with allergen. To assess the role of CCR3 in the recruitment of cells to the lung and into the airways, we examined BAL fluid and lung sections from CCR $3^{-/-}$mice and WT controls following inhalation challenge with OVA. As we previously showed (24), few eosinophils were present in BAL fluid from shamsensitized WT mice following OVA challenge, whereas eosinophil numbers were markedly increased in BAL fluid from OVA-sensitized WT mice (Figure 4a). In contrast, no eosinophils were detected in BAL fluid from sham-sensitized $C C R 3^{-/-}$mice, and eosinophil numbers were severely reduced ( $89 \%)$ in BAL fluid from OVAsensitized CCR3 $3^{-/-}$mice (Figure 4a). Small numbers of neutrophils were present in BAL fluid from sham-sensitized WT mice and CCR3 $3^{--}$mice. Neutrophil numbers were increased in BAL fluid from both OVA-sensitized WT mice and CCR3-- mice (Figure 4a). Increase in CCR3 ${ }^{-/-}$mice was less than that observed in OVA-sensitized WT controls, but the difference was not statistically significant (Figure 4a). The number of T lymphocytes in the BAL fluid of WT and CCR3-/- mice was equivalent and did not differ between mice sensitized with saline and those sensitized with OVA (Figure 4a).

The failure of eosinophil recruitment into the airway in $\mathrm{CCR} 3^{-/}$mice may have resulted from failure to mobilize eosinophils from the blood into lung tissue and/or from failure to mobilize eosinophils from the lung tissue into the airway. Following OVA challenge, few eosinophils were present in the lung parenchyma of sham-sensitized WT mice. The numbers of infiltrating eosinophils were markedly increased in OVA-sensitized WT mice (Figure 4b). Eosinophils were rarely detected in lungs from sham-sensitized CCR3-/- mice. The number of infiltrating eosinophils increased in OVA-sensitized CCR3-- mice, but significantly less than that in OVA-sensitized WT control (Figure 4b). Taken together, these data suggest that CCR3 is essential for eosinophil recruitment to the lung following antigen sensitization and challenge.

Mast cells are considered important effector cells in the allergic airway response (42). We examined mast cells in the trachea following inhalation challenge with OVA in WT mice and CCR3-- mice. Mast cell numbers were similar in sham-sensitized CCR3-/- mice $(6 \pm 0.6$ mast cells/trachea ring) and WT controls $(8.3 \pm 2.2$ mast cells/trachea ring). Mast cell numbers did not increase in OVA-sensitized CCR3-- mice $(7.5 \pm 0.9$ mast cells/trachea ring) or WT controls ( $10.8 \pm 3.3$ mast cells/trachea ring). There was no evidence of degranulation either in
WT or in CCR3-/- mice (Figure 4c). Mast cells were localized in the submucosa, but not intraepithelially, in both $\mathrm{CCR3}^{-/-}$mice and WT controls (Figure 4c).

CCR3 ${ }^{-1-}$ mice epicutaneously sensitized with OVA do not develop AHR to Mch following inhalation challenge with allergen. AHR to inhaled antigen develops in epicutaneously sensitized mice (24). Inhalation of a single dose of OVA by WT mice epicutaneously sensitized with OVA resulted 24 hours later in a significant increase in AHR to Mch compared with sham-sensi-

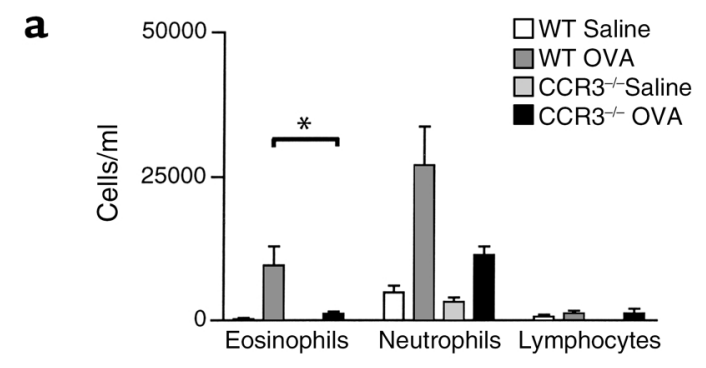

b

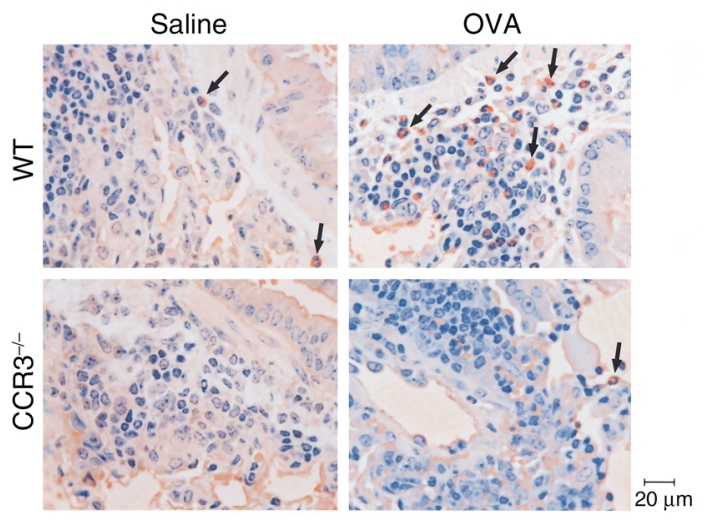

c

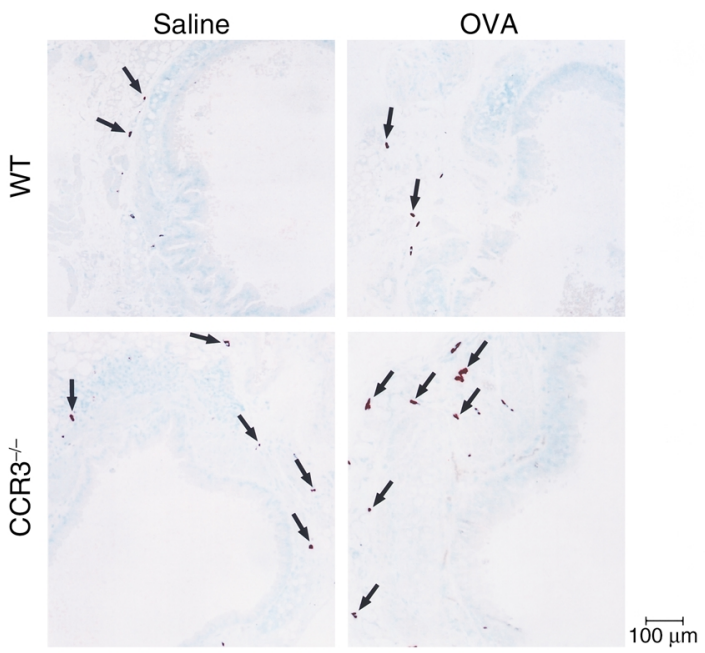

Figure 4

$C C R 3^{-/-}$mice epicutaneously sensitized with OVA do not develop BAL or lung tissue eosinophilia following inhalation challenge with allergen. (a) Eosinophils, neutrophils, and lymphocytes in BAL fluid were counted. The bars represent the mean ( $n=6-7$ animals per group). ${ }^{*} P<0.01$. (b) Lung sections taken 24 hours after a single exposure of inhaled 1\% OVA and stained with Congo red dye, which stains eosinophils orange (arrows). (c) Trachae sections taken 24 hours after a single exposure of inhaled 1\% OVA and stained with a solution containing naphthol AS-D chloroacetate to identify mast cells (arrows). 


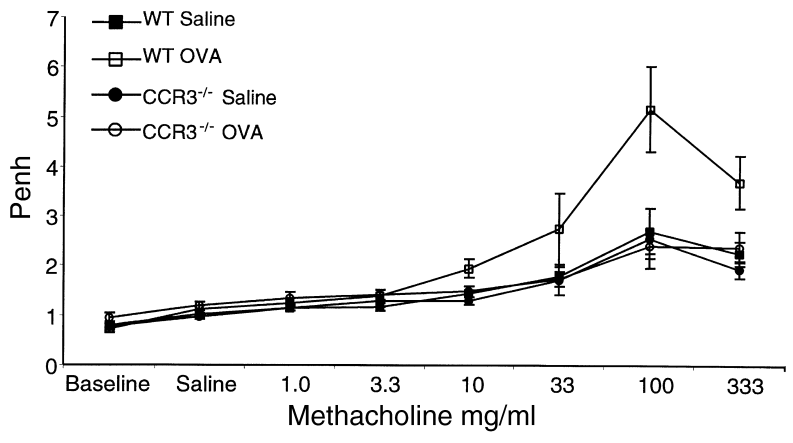

Figure 5

CCR3-1- mice epicutaneously sensitized with OVA do not develop AHR to Mch following inhalation challenge with allergen. AHR was measured by whole body plethysmography in conscious $\mathrm{CCR} 3^{-/-}$mice and WT controls following inhalation challenge with OVA. Penh, an index of airway obstruction, was calculated from the box pressure/time wave after aerosolization of increasing doses of Mch. Numerically higher values of Penh are indicative of increased airway obstruction. Data represents mean Penh values $\pm \operatorname{SEM}(n \geq 6$ mice) .

tized WT controls $(P=0.0002)$ as measured by whole body plethysmography during the challenge and calculated by Penh. In contrast, OVA-sensitized CCR3-1mice failed to exhibit AHR compared with OVA-sensitized WT controls $(P<0.0002)$ and had a similar response to Mch as sham-sensitized WT and CCR3-1mice $(P>0.05)$ (Figure 5).

The Th2 cytokine IL- 4 has been implicated in the development of AHR (42). There was negligible expression of IL-4 mRNA in lungs from sham-sensitized WT and $C C R 3^{-/-}$mice challenged with inhaled OVA. There was comparably increased expression of IL-4 mRNA in lungs from both OVA-sensitized WT and CCR3 $3^{-1-}$ mice challenged with inhaled OVA (Figure 6). Furthermore, mRNA expression of the Th2-selected genes IL-5 and GATA-3 was equivalent in CCR3-/- mice and WT controls (data not shown). These results suggest that the absent AHR in CCR3 $3^{--}$mice is not due to a defect in Th2 cytokine expression in the lungs.

\section{Discussion}

The present study demonstrates that eosinophils are absent from the skin of CCR3-/- mice and fail to infiltrate their skin following repeated epicutaneous sensitization with OVA. Recruitment of eosinophils to lung parenchyma and into airways following OVA antigen inhalation challenge is also severely impaired in epicutaneously sensitized CCR3 $3^{-/}$mice. Furthermore, epicutaneously sensitized mice fail to develop AHR.

Eosinophils and their product MBP were virtually absent from both sham and OVA-sensitized skin of CCR3 $3^{-/}$mice (Figure 1, a and b). Eosinophils were also severely decreased in the lung and BAL fluid of epicutaneously sensitized CCR3-- mice challenged with inhalation of OVA (Figure 4, a and b). However, lack of CCR3 does not affect eosinophil migration in general. Baseline eosinophil numbers in thymus and lung were normal in CCR3 ${ }^{-/-}$mice (27). Furthermore, eosinophils from CCR3-- mice instilled into the trachea of WT mice migrate normally to draining lymph nodes (43). These results suggest that CCR 3 plays an essential role in eosinophil recruitment to the skin and the lung.

Eotaxin is an important ligand for CCR3. There is conflicting data regarding the role of eotaxin in eosinophil recruitment to the lung following antigen challenge. In one study, eosinophil numbers in BAL fluid of eotaxin-null mice were reduced by $70 \% 18$ hours, but not 48 hours, after challenge (20). In another study, eosinophil numbers in BAL fluid of eotaxinnull mice were normal 18 hours after allergen challenge (44). These data suggest that eotaxin could be redundant in eosinophil recruitment to the lung following antigen challenge. Other CCR3 ligands, such as MCP-3 or RANTES, may also be important for eosinophil recruitment to the lung.

In addition to CCR3, eosinophils may also express CCR1 (45), IL-8 receptor (46), and possibly other unidentified chemokine receptors (47). CCR1 is a receptor for RANTES and MIP-1 $\alpha$. Tissue expression of both of these chemokines is increased in $\mathrm{AD}$ and asthma $(25,48-52)$. Nevertheless, our data suggest that CCR1 and IL-8 receptor play a minor role, if any, in eosinophil recruitment into inflamed skin and lung.

A recent study has shown that infusion of eotaxin results in rapid blood eosinophilia and synergizes with IL-5 in eosinophil mobilization (53), suggesting a role for eotaxin in mobilization of eosinophils from bone marrow. Examination of the physiologic role of eotaxin in eosinophil mobilization using eotaxin-deficient mice has yielded conflicting results. One line of eotaxin-deficient mice showed decreased blood eosinophil counts (20), and the other line showed normal blood eosinophil counts (44). Blood eosinophil counts were comparable in CCR3 $3^{--}$and WT controls. Given the fact that CCR3 is the only known receptor for eotaxin, these results suggest that eotaxin may not play an important role in the mobilization of eosinophils from the bone marrow in our model. Furthermore, CCR3 deficiency did not interfere with the production of the IL-5 (Fig-

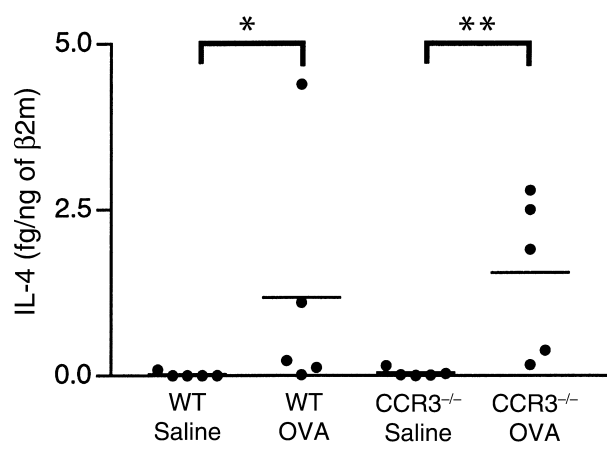

Figure 6

The Th2 cytokine is expressed normally in lungs of epicutaneously sensitized $\mathrm{CCR}^{-/-}$mice following allergen inhalation challenge. IL-4 mRNA levels were normalized to $\beta 2$-microglobulin. The bars represent the mean $\left(n=5\right.$ animals per group). ${ }^{*} P<0.05 ;{ }^{*} P<0.01$. 
ure $3 b$ ), which is well established as a major eosinophil maturation- and mobilization-inducing cytokine.

Mast cells have been reported to express CCR3 as well as other chemokine receptors, which include CCR1, CCR2, CCR5, and CXCR4 $(54,55)$. Sham-sensitized skin of $C C R 3^{-/-}$mice had comparable numbers of mast cells as skin from WT controls (data not shown). This suggests that CCR3 is either redundant or not important for mast cell trafficking to skin. There was no significant increase in mast cell numbers in OVA-sensitized skin sites of WT mice of the 129/BALB/c background or in their CCR3-/- littermates (data not shown). Therefore the role of CCR3 in the recruitment of mast cells into inflamed skin in our model cannot be determined from the present study.

Th2 but not Th1 cells express CCR3 (15). Production of IL-4 (Figure 3a) and IL-5 (Figure 3b) by splenocytes stimulated with OVA were normal, and serum levels of OVA-specific IgE (Figure3c) were normal in CCR3-/mice. These results suggest that CCR3 may not be important for the differentiation of Th2 cells. IL-4 can be expressed by $T$ cells, mast cells, and eosinophils (56, 57). IL-4 mRNA expression in OVA-sensitized skin is absent in TCR $\alpha \beta^{-/-}$mice (37), but normal in mast cell-deficient $\left(W / W^{v}\right)$ mice (58), suggesting that T cells are the major source of skin IL-4 in our model. Expression of the Th2 cytokine, IL-4, in OVA-sensitized skin was comparable in CCR3-/- mice and WT controls (Figure $2 \mathrm{~b}$ ), suggesting that CCR3 does not play an important role in the recruitment of Th2 cells to sites of allergic inflammation. In support of this notion is our finding of comparable mRNA expression of the Th2selective genes, IL-4 (Figure 6), IL-5, and GATA-3 (data not shown) in the antigen-challenged lungs of epicutaneously sensitized CCR $3^{-/-}$mice and WT controls.

Our present observation that eosinophil recruitment to lung and AHR are both severely diminished in epicutaneously sensitized mice (Figure $4 \mathrm{~b}$ and Figure 5) does not necessarily mean that the two are causally related. In mice intraperitoneally sensitized with antigen, AHR and lung eosinophilia are often, although not always (59), dissociated, suggesting that eosinophils may not be important for AHR in this model $(60,61)$. We have recently observed that $\mathrm{AHR}$ is enhanced, while eosinophil recruitment to lung and BAL is significantly diminished, in $\mathrm{CCR}^{-/-}$mice intraperitoneally sensitized with OVA antigen (27). The different results we obtained in the epicutaneous sensitization and intraperitoneal sensitization models suggest that the mechanisms of development of AHR may differ with different routes of immunization. We have found that mast cells are mobilized to the airway epithelium in intraperitoneally sensitized WT mice (27), but not in epicutaneously sensitized WT mice, following inhalation challenge (Figure 4c). Mast cell mobilization into airway epithelium is significantly increased in intraperitoneally sensitized CCR3-/mice (27), but remains absent in epicutaneously sensitized CCR3-/- mice (Figure 4c). These findings suggest that CCR3-independent mobilization of mast cells into the airway epithelium is an important player in AHR in the intraperitoneal sensitization model. In the epicutaneous sensitization model, in the absence of mast cells in airway epithelium CCR3-dependent recruitment of eosinophils may become an important player in AHR. The finding that mast cells are mobilized to the epithelium in intraperitoneally sensitized mice, but not in epicutaneously sensitized mice, suggests that the immune response may differ between the two models. As a matter of fact, the epicutaneous model is more of a predominant Th2 response $(24,62)$, while the intraperitoneal model has a stronger Th1 component (63). Th1 cells in the latter may induce the production of chemokines other than CCR3 ligands (e.g., SDF), which modulate eosinophil and mast cell trafficking. Further work is needed to test this hypothesis.

Taken together, our data suggest CCR3 is essential for eosinophil recruitment to the skin and the lung and for AHR in response to antigen inhalation in epicutaneously sensitized mice. Targeting CCR3 may offer a possible therapy for $\mathrm{AD}$ and allergic asthma.

\section{Acknowledgments}

This work was supported by United States Public Health Service (USPHS) grant AR47417 (to R.S. Geha). W. Ma was supported by USPHS grant T32-AI07512.

1. Leung, D.Y.M. 1995. Atopic dermatitis: the skin as a window into the pathogenesis of chronic allergic disease. J. Allergy Clin. Immunol. 96:312-319.

2. Leung, D.Y.M. 1992. Immunopathology of atopic dermatitis. Springer Semin. Immunopathol. 13:427-440.

3. Thepen, T., et al. 1996. Biphasic response against aeroallergen in atopic dermatitis showing a switch from an initial Th2 response to a Th1 response in situ: an immunocytochemical study. J. Allergy Clin. Immunol. 97:828-837.

4. Leung, D.Y. 2000. Atopic dermatitis: new insights and opportunities for therapeutic intervention. J. Allergy Clin. Immunol. 105:860-876.

5. Gleich, G.J. 2000. Mechanisms of eosinophil-associated inflammation. J. Allergy Clin. Immunol. 105:651-663.

6. van de Rijn, M., et al. 1998. A murine model of allergic rhinitis: studies on the role of IgE in pathogenesis and analysis of the eosinophil influx elicited by allergen and eotaxin. J. Allergy Clin. Immunol. 102:65-74.

7. Rajka, G. 1975. Major problems in dermatology: atopic dermatitis. Volume 3. W.B. Saunders Co., Philadelphia, Pennsylvania, USA. 42 pp.

8. Wedi, B., Raap, U., Lewrick, H., and Kapp, A.. 1997. Delayed eosinophil programmed cell death in vitro: a common feature of inhalant allergy and extrinsic and intrinsic atopic dermatitis. J. Allergy Clin. Immunol. 100:536-543.

9. Leiferman, K.M. 1994. Eosinophils in atopic dermatitis. J. Allergy Clin. Immunol. 94:1310-1317.

10. Leiferman, K.M., et al. 1985. Dermal deposition of eosinophil-granule major basic protein in atopic dermatitis: comparison with onchocerciasis. N. Engl. J. Med. 313:282-285.

11. Ott, N.L., et al. 1994. Assessment of eosinophil and neutrophil participation in atopic dermatitis: comparison with the IgE-mediated latephase reaction. J. Allergy Clin. Immunol. 94:120-128.

12. Sallusto, F., Mackay, C.R., and Lanzavecchia, A. 2000. The role of chemokine receptors in primary, effector, and memory immune responses. Annu. Rev. Immunol. 18:593-620.

13. Ponath, P.D., et al. 1996. Molecular cloning and characterization of a human eotaxin receptor expressed selectively on eosinophils. J. Exp. Med. 183:2349-2354.

14. Ochi, H., et al. 1999. T helper cell type 2 cytokine-mediated comitogenic responses and CCR3 expression during differentiation of human mast cells in vitro. J. Exp. Med. 190:267-280.

15. Sallusto, F., Mackay, C.R., and Lanzavecchia, A. 1997. Selective expression of the eotaxin receptor CCR3 by human T helper 2 cells. Science. 277:2005-2007.

16. Homey, B., and Zlotnik, A. 1999. Chemokines in allergy. Curr. Opin. Immunol. 11:626-634.

17. Ying, S., et al. 1997. Enhanced expression of eotaxin and CCR3 mRNA 
and protein in atopic asthma. Association with airway hyperresponsiveness and predominant co-localization of eotaxin mRNA to bronchial epithelial and endothelial cells. Eur. J. Immunol. 27:3507-3516

18. Stafford, S., et al. 1997. Monocyte chemotactic protein-3 (MCP3)/fibro-blast-induced cytokine (FIC) in eosinophilic inflammation of the airways and the inhibitory effects of an anti-MCP-3/FIC antibody. J. Immunol. 158:4953-4960.

19. Gonzalo, J.A., et al. 1998. The coordinated action of CC chemokines in the lung orchestrates allergic inflammation and airway hyperresponsiveness. J. Exp. Med. 188:157-167.

20. Rothenberg, M.E., MacLean, J.A., Pearlman, E., Luster, A.D., and Leder, P. 1997. Targeted disruption of the chemokine eotaxin partially reduces antigen-induced tissue eosinophilia. J. Exp. Med. 185:785-790.

21. Ying, S., Taborda-Barata, L., Meng, Q., Humbert, M., and Kay, A.B. 1995. The kinetics of allergen-induced transcription of messenger RNA for monocyte chemotactic protein-3 and RANTES in the skin of human atopic subjects: relationship to eosinophil, $T$ cell, and macrophage recruitment. J. Exp. Med. 181:2153-2159.

22. Schroder, J.M., Noso, N., Sticherling, M., and Christophers, E. 1996. Role of eosinophil-chemotactic C-C chemokines in cutaneous inflammation. J. Leukoc. Biol. 59:1-5.

23. Yawalkar, N., et al. 1999. Enhanced expression of eotaxin and CCR3 in atopic dermatitis. J. Invest. Dermatol. 113:43-48.

24. Spergel, J., et al. 1998. Epicutaneous sensitization with protein antigen induces localized allergic dermatitis and hyperresponsiveness to metacholine after single exposure to aerosolized antigen in mice. J. Clin. Invest. 101:1614-1622.

25. Spergel, J.M., Mizoguchi, E., Oettgen, H., Bhan, A.K., and Geha, R.S. 1999. Roles of TH1 and TH2 cytokines in a murine model of allergic dermatitis. J. Clin. Invest. 103:1103-1111.

26. Eliasson, A.H., Phillips, Y.Y., Rajagopal, K.R., and Howard, R.S. 1992 Sensitivity and specificity of bronchial provocation testing. An evaluation of four techniques in exercise-induced bronchospasm. Chest. 102:347-355.

27. Humbles, A.A., et al. 2001. The murine CCR3 receptor regulates both the role of eosinophils and mast cells in allergen-induced airway inflammation and hyperresponsiveness. Proc. Natl. Acad. Sci. USA. 99:1479-1484.

28. Sawada, K., et al. 1997. The expression of murine cutaneous late phase reaction requires both $\mathrm{IgE}$ antibodies and CD4 T cells. Clin. Exp. Aller gy. 27:225-231.

29. Friend, D.S., Gurish, M.F., Austen, K.F., Hunt, J., and Stevens, R.L. 2000 Senescent jejunal mast cells and eosinophils in the mouse preferentially translocate to the spleen and draining lymph node, respectively, during the recovery phase of helminth infection. J. Immunol. 165:344-352.

30. Mombaerts, P., et al. 1993. Spontaneous development of inflammatory bowel disease in T cell receptor mutant mice. Cell. 75:275-282.

31. Lee, J.J., et al. 1997. Interleukin-5 expression in the lung epithelium of transgenic mice leads to pulmonary changes pathognomonic of asthma. J. Exp. Med. 185:2143-2156.

32. Shire, D. 1993. An invitation to an open exchange of reagents and information useful for the measurements of cytokine mRNA levels by PCR Eur. Cytokine Netw. 4:161-162.

33. Shire, D., and Legoux, P. 1995. Gene expression analysis using quantitative reverse transcription polymerase chain reaction and multispecific internal control. Humana Press Inc., Totowa, New Jersey, USA.

34. Colley, D. 1972. Schistosoma mansoni: eosinophilia and the development of lymphocyte blastogenesis in response to soluble egg antigen in inbred mice. Exp. Parasitol. 32:520-526.

35. Hamelmann, E., et al. 1997. Noninvasive measurement of airway responsiveness in allergic mice using barometric plethysmography. Am. J. Respir. Crit. Care Med. 156:766-775.

36. Peters, M.S., Schroeter, A.L., and Gleich, G.J. 1983. Immunofluorescence identification of eosinophil granule major basic protein in the flame figures of Wells' syndrome. Br. J. Dermatol. 109:141-148.

37. Woodward, A.L., et al. 2001. An obligate role for T-cell receptor alphabeta $+\mathrm{T}$ cells but not $\mathrm{T}$-cell receptor gammadelta $+\mathrm{T}$ cells, B cells, or CD40/CD40L interactions in a mouse model of atopic dermatitis. $J$. Allergy Clin. Immunol. 107:359-366.

38. Jinquan, T., Quan, S., Feili, G., Larsen, C.G, and Thestrup-Pedersen, K. 1999. Eotaxin activates $T$ cells to chemotaxis and adhesion only if induced to express CCR3 by IL-2 together with IL-4. J. Immunol. 162:4285-4292.

39. Metcalfe, D., Costa, J., and Burd, P. 1992. Mast cells and basophils. Raven
Press Ltd., New York, New York, USA. 709-723.

40. Romagnani, P., et al. 1999. Tryptase-chymase double-positive human mast cells express the eotaxin receptor CCR3 and are attracted by CCR3-binding chemokines. Am. J. Pathol. 155:1195-1204.

41. de Paulis, A., et al. 2001. Expression of the chemokine receptor CCR3 on human mast cells. Int. Arch. Allergy Immunol. 124:146-150.

42. Wills-Karp, M. 1999. Immunologic basis of antigen-induced airway hyperresponsiveness. Annu. Rev. Immunol. 17:255-281.

43. Shi, H.Z., Humbles, A., Gerard, C., Jin, Z., and Weller, P.F. 2000. Lymph node trafficking and antigen presentation by endobronchial eosinophils. J. Clin. Invest. 105:945-953.

44. Yang, Y., Loy, J., Ryseck, R.P., Carrasco, D., and Bravo, R. 1998. Antigeninduced eosinophilic lung inflammation develops in mice deficient in chemokine eotaxin. Blood. 92:3912-3923.

45. Sabroe, I., et al. 1999. Differential regulation of eosinophil chemokine signaling via CCR3 and non-CCR3 pathways. J. Immunol. 162:2946-2955

46. Erger, R.A., and Casale, T.B. 1995. Interleukin- 8 is a potent mediator of eosinophil chemotaxis through endothelium and epithelium. Am. J. Physiol. 268:L117-L122.

47. Bochner, B.S., et al. 1999. Macrophage-derived chemokine induces human eosinophil chemotaxis in a CC chemokine receptor 3-and CC chemokine receptor 4-independent manner. J. Allergy Clin. Immunol. 103:527-532.

48. Hatano, Y., Katagiri, K., and Takayasu, S. 1999. Increased levels in vivo of mRNAs for IL-8 and macrophage inflammatory protein-1 alpha (MIP-1 alpha), but not of RANTES mRNA in peripheral blood mononuclear cells of patients with atopic dermatitis (AD). Clin. Exp. Immunol. 117:237-243.

49. Ordonez, C.L., Shaughnessy, T.E., Matthay, M.A., and Fahy, J.V. 2000. Increased neutrophil numbers and IL-8 levels in airway secretions in acute severe asthma: clinical and biologic significance. Am. J. Respir. Crit. Care Med. 161:1185-1190.

50. Hoshi, H., et al. 1995. IL-5, IL-8 and GM-CSF immunostaining of sputum cells in bronchial asthma and chronic bronchitis. Clin. Exp. Allergy. 25:720-728.

51. Alam, R., et al. 1996. Increased MCP-1, RANTES, and MIP-1 alpha in bronchoalveolar lavage fluid of allergic asthmatic patients. Am. J. Respir. Crit. Care Med. 153:1398-1404.

52. Holgate, S.T., et al. 1997. Release of RANTES, MIP-1 alpha, and MCP1 into asthmatic airways following endobronchial allergen challenge. Am. J. Respir. Crit. Care Med. 156:1377-1383.

53. Palframan, R.T., Collins, P.D., Williams, T.J., and Rankin, S.M. 1998 Eotaxin induces a rapid release of eosinophils and their progenitors from the bone marrow. Blood. 91:2240-2248.

54. Juremalm, M., et al. 2000. The chemokine receptor CXCR4 is expressed within the mast cell lineage and its ligand stromal cell-derived factor1alpha acts as a mast cell chemotaxin. Eur. J. Immunol. 30:3614-3622.

55. Oliveira, S.H., and Lukacs, N.W. 2001. Stem cell factor and IgE-stimulated murine mast cells produce chemokines (CCL2, CCL17, CCL22) and express chemokine receptors. Inflamm. Res. 50:168-174.

56. Nouri-Aria, K.T., et al. 2000. Cytokine expression during allergeninduced late nasal responses: IL-4 and IL-5 mRNA is expressed early (at 6 h) predominantly by eosinophils. Clin. Exp. Allergy. 30:1709-1716

57. Nakajima, H., Gleich, G.J., and Kita, H. 1996. Constitutive production of IL- 4 and IL-10 and stimulated production of IL- 8 by normal peripheral blood eosinophils. J. Immunol. 156:4859-4866.

58. Alenius, H., et al. 2001. Mast cells regulate IFN- $\gamma$ expression in the skin and circulating IgE levels in allergen induced skin inflammation. J. Allergy Clin. Immunol. 109:106-113.

59. Foster, P., Hogan, S., Ramsay, A., Matthaei, K., and Young, I. 1996. Interleukin 5 deficiency abolishes eosinophilia, airways hyperreactivity and lung damage in a mouse asthma model. J. Exp. Med. 183:195-201.

60. Corry, D.B., et al. 1996. Interleukin 4, but not interleukin 5 or eosinophils, is required in a murine model of acute airway hyperreactivity. J. Exp. Med. 183:109-117.

61. Walter, D.M., et al. 2001. Critical role for IL-13 in the development of allergen-induced airway hyperreactivity. J. Immunol. 167:4668-4675.

62. Herrick, C.A., MacLeod, H., Glusac, E., Tigelaar, R.E., and Bottomly, K. 2000. Th2 responses induced by epicutaneous or inhalational protein exposure are differentially dependent on IL-4. J. Clin. Invest. 105:765-775.

63. Mattes, J., et al. 2001. IL-13 induces airways hyperreactivity independently of the IL-4R alpha chain in the allergic lung. J. Immunol. 167:1683-1692 